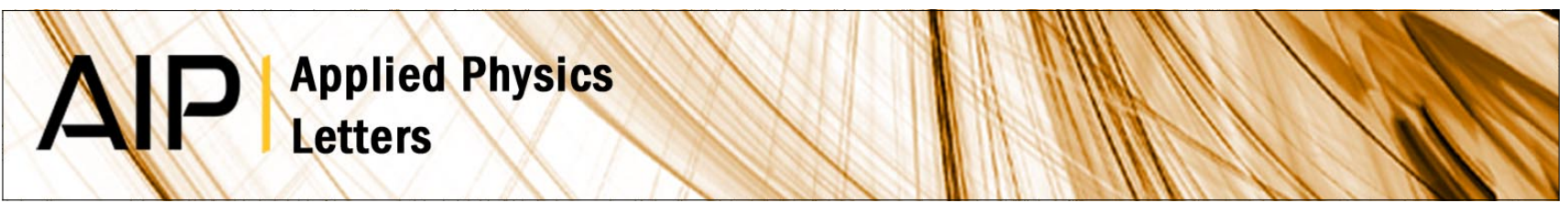

\title{
Compact tunable sub-terahertz oscillators based on Josephson junctions
}

Fengbin Song, Franz Müller, Thomas Scheller, Alexei Semenov, Ming He et al.

Citation: Appl. Phys. Lett. 98, 142506 (2011); doi: 10.1063/1.3576910

View online: http://dx.doi.org/10.1063/1.3576910

View Table of Contents: http://apl.aip.org/resource/1/APPLAB/v98/i14

Published by the American Institute of Physics.

Additional information on Appl. Phys. Lett.

Journal Homepage: http://apl.aip.org/

Journal Information: http://apl.aip.org/about/about_the_journal

Top downloads: http://apl.aip.org/features/most_downloaded

Information for Authors: http://apl.aip.org/authors

\section{ADVERTISEMENT}

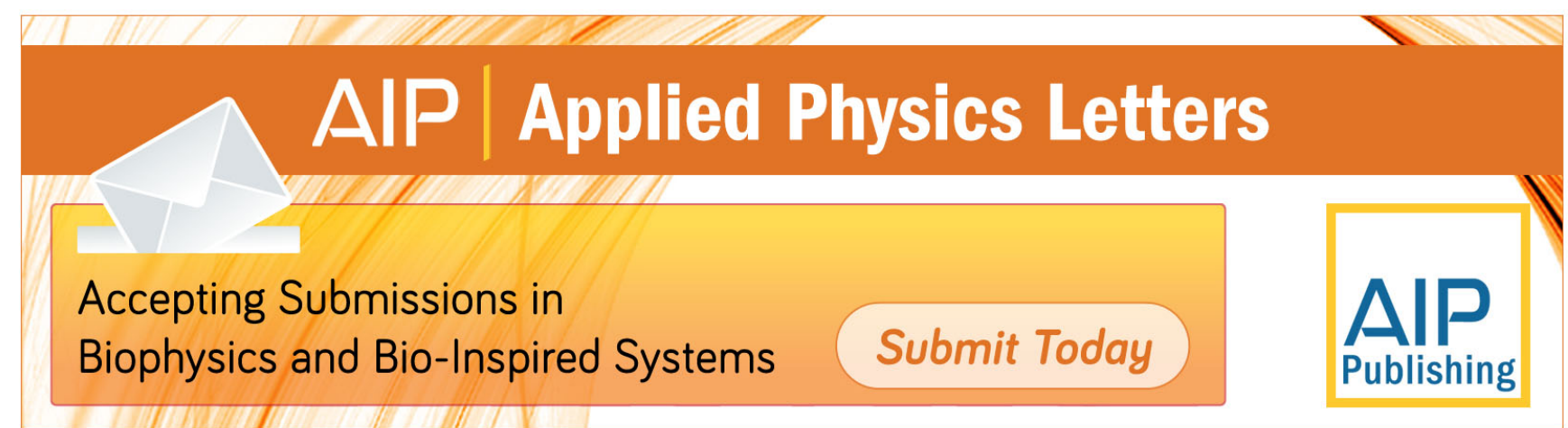




\title{
Compact tunable sub-terahertz oscillators based on Josephson junctions
}

\author{
Fengbin Song (宋凤斌), ${ }^{1,4}$ Franz Müller, ${ }^{2}$ Thomas Scheller, ${ }^{2}$ Alexei Semenov, ${ }^{3}$ Ming He, ${ }^{4}$ \\ Lan Fang, ${ }^{4}$ Heinz-Wilhelm Hübers, ${ }^{3}$ and Alexander M. Klushin ${ }^{1,5, a)}$ \\ ${ }^{1}$ Institute of Bio- and Nanosystems and JARA-Fundamentals of Future Information Technology, \\ Forschungszentrum Jülich, D-52425 Jülich, Germany \\ ${ }^{2}$ Physikalisch-Technische Bundesanstalt, 38116 Braunschweig, Germany \\ ${ }^{3}$ Institute of Planetary Research, German Aerospace Centre (DLR), 12489 Berlin, Germany \\ ${ }^{4}$ Department of Electronics, Nankai University, 300071 Tianjin, People's Republic of China \\ ${ }^{5}$ Institute for Physics of Microstructures RAS, 603950 Nizhny Novgorod, Russia
}

(Received 18 February 2011; accepted 17 March 2011; published online 8 April 2011)

\begin{abstract}
Essential applications of terahertz technology are urgently in need of compact, tunable solid-state continuous wave radiation sources. However, no satisfactory solution is yet available for the frequency range of up to approximately $1.0 \mathrm{THz}$. Here, we present coherent radiation from large series arrays of Josephson junctions between 0.1 and $0.25 \mathrm{THz}$ with off-chip radiation power of $7 \mu \mathrm{W}$. Niobium junctions oscillate at $4.2 \mathrm{~K}$ and the detection has been done at room temperature. The well-known obstacle to impedance matching is overcome by utilizing the excited resonances in the junction substrates serving as dielectric resonator antennae. (C) 2011 American Institute of Physics. [doi:10.1063/1.3576910]
\end{abstract}

Josephson junctions have been expected to be terahertz oscillators for about half a century, ever since Josephson discovered the effects named after him. ${ }^{1}$ The ac Josephson effect causes Josephson junctions to be by nature electrically pumped high-frequency oscillators. Their radiation frequency is tunable with $1 \mathrm{mV}$ of dc bias corresponding to $483.6 \mathrm{GHz}^{2}$ However, the relatively small radiation power coupled off-chip, normally on the nanowatt level, ${ }^{3,4}$ is the major remaining obstacle to practical applications. This obstacle can be removed by synchronizing large arrays of Josephson junctions and improving impedance matching of the junctions to the open space. ${ }^{5}$

Dielectric resonator antennae have been extensively investigated for modern wireless communications. These resonators are inherently suitable for high-frequency applications, because of the elimination of alternating current loss in the metal. In our case, their resonant modes can also be utilized for synchronizing Josephson junctions in a large array. We discovered that various dielectric substrates for depositing integrated Josephson junction arrays are suitable for this task. $^{6,7}$

When Josephson junctions are strongly coupled to a resonator, self-induced steps, and non-Josephson oscillation can be observed. ${ }^{8}$ The non-Josephson radiation was first detected in 1966 from a single point-contact Josephson junction coupled to a rectangular cavity. ${ }^{9}$ Further theoretical investigations were performed by Auracher and Van Duzer ${ }^{10}$ and experiments by Kanter. ${ }^{11}$ The parametric negativeresistance effect generated by the quantum-phase-dependent inductance of the Josephson junction $L_{\mathrm{J}}$, was considered to be the explanation of the self-induced steps and of the nonJosephson oscillation. The standard parametric amplifier theory applies if the impedance of the intrinsic inductance $L_{\mathrm{J}}$ for alternating currents is quite large compared to the external impedance. ${ }^{12}$ Otherwise, the supercurrent path becomes dominant and its impedance could be real and negative for the frequencies near to the resonant frequencies of

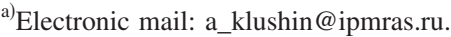

external circuits. In the latter case, the junctions will normally be underdamped with hysteretic current-voltage $(I-V)$ characteristics. $^{8,11}$ The negative-resistance simply means the junction serves as an oscillator emitting power to the external circuits. Once the junctions are coupled to a resonator, the current induced in it will be excited and this induced current may feed back to the junctions. Therefore, self-induced steps emerge around the bias voltages $V$ corresponding to the resonant mode frequencies $f_{\mathrm{r}}$. The oscillation induced in the resonator can be coupled into the open space and then detected as the non-Josephson oscillation.

In this letter, we present coherent tunable radiation of a large series junction array, carefully designed to excite resonant modes of the array substrate serving as a dielectric resonator antenna. The detected radiation covered the frequency range from about $0.10 \mathrm{THz}$ up to more than $0.25 \mathrm{THz}$, with maximum radiation power up to $7 \mu \mathrm{W}$ detected at room temperature around $0.143 \mathrm{THz}$. We documented that the radiation frequency is shifted from the Josephson oscillation frequency $f_{\mathrm{J}}$ to the resonant frequency $f_{\mathrm{r}}$, which originated the so-called non-Josephson oscillation. ${ }^{8}$ We see promise in coupling Josephson junction arrays to modified substrates to extend this coherent radiation into the sub- $\mathrm{THz}$ range with higher power emitted.

In our experiment, recently developed $\mathrm{Nb}-\mathrm{Si}$ barrier junctions ${ }^{13,14}$ were adopted to fabricate a series array including $N=6972$ junctions on silicon substrate with a dielectric constant of 11.9 and dimensions of $10 \times 10 \times 0.38 \mathrm{~mm}^{3}$. The overall view of our sample is shown in the top left corner of Fig. 1. The series array contained seven meandering subarrays of 996 junctions each placed in groups of 332. The distance between each subarray is $714 \mu \mathrm{m}$; such design excites the resonant mode of the substrate around $0.15 \mathrm{THz}$. Special thin-film conductor tabs allowed for an independent dc bias and radiation measurement of each subarray and of selected groups. The junctions were fabricated with lateral dimensions of $8 \times 8 \mu \mathrm{m}^{2}$, and the distance between them was $7 \mu \mathrm{m}$. The critical current of a single junction $I_{\mathrm{c}}$ was about $2 \mathrm{~mA}$, the corresponding critical current density $J_{\mathrm{c}}$ 


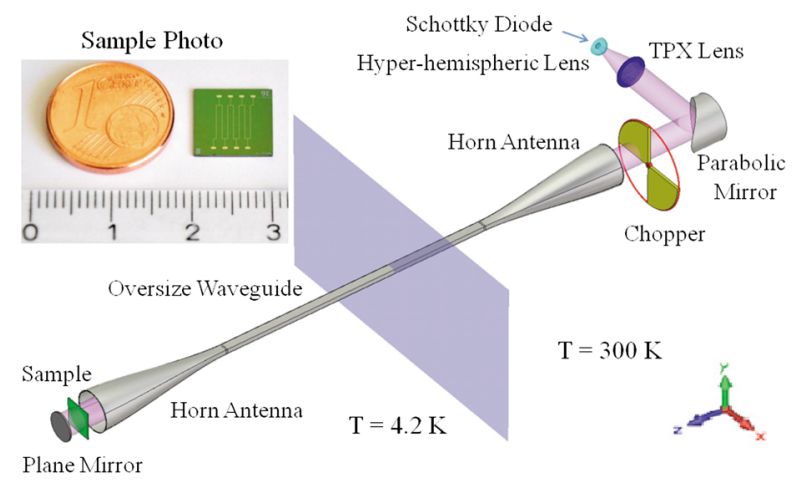

FIG. 1. (Color online) Measurement setup for sub-THz radiation. The left inset shows the sample photo compared to an eurocent coin.

$=3.1 \mathrm{~A} / \mathrm{cm}^{2}$, and the normal resistance $R_{n}=0.118 \Omega$. Therefore, for each junction the average characteristic voltage $V_{\mathrm{c}}$ was $0.226 \mathrm{mV}$ and the characteristic frequency 114 $\mathrm{GHz}$.

The schematic view of the measurement system is shown in Fig. 1. The non-Josephson oscillation pumped by the current-biased series array was coupled through the substrate into the open space. An external quasioptical resonator consisting of a horn antenna and a plane mirror ${ }^{6}$ was introduced to collect the radiation and then transmit it through an oversized waveguide into the ultrawideband sub-THz Schottky-diode detection system working at room temperature. $^{15}$

A typical example of the measured $I-V$ curve of the junction array with a series of the self-induced steps is shown in Fig. 2. By adjusting the distance between the plane mirror and our sample, the position of the horn antenna and the polarization of the electric field inside the quasioptical resonator, several radiation peaks were observed on these steps, as also shown in Fig. 2. Since hysteresis was observed in the $I-V$ curve, some radiation peaks presented different characteristics for the bias current sweeping up and down. The amplitude of each peak could be optimized by adjusting the above parameters. For example, the amplitude of peaks at frequencies higher than $0.15 \mathrm{THz}$ was enhanced [Fig. 2(b)] by decreasing the distance between the plane mirror and our sample. The maximum radiation power of about $7 \mu \mathrm{W}$ was detected at $0.143 \mathrm{THz}$. The total emitted power should be much larger, as only a fraction of the radiation power couples to the detection system.

We measured the exact radiation frequency $f$ of the peak observed around $0.143 \mathrm{THz}$ with a spectrum analyzer using a heterodyne receiver, which included a WR-6 (110-170 GHz) harmonic mixer pumped at frequencies from $68 \mathrm{GHz}$ to 78 GHz. As shown in Fig. 3(a), the radiation frequency $f$ was tunable by adjusting the bias voltage $V$ across the series junction array. However, compared with the Josephson oscillation frequencies $f_{J}=V / N \Phi_{0}$, where the $\Phi_{0}$ is the flux quantum, the observed radiation frequencies $f$ were shifted to the central resonant frequency $f_{\mathrm{r}}$ of the substrate cavity [Fig. 3(a)], thus representing the non-Josephson oscillation. ${ }^{8}$ The Josephson frequencies $f_{\mathrm{J}}$ on the three arrow-marked bias points in Fig. 3(a) are equal to $142.82 \mathrm{GHz}, 143.30 \mathrm{GHz}$, and $143.58 \mathrm{GHz}$, while the observed radiation frequencies are $142.94 \mathrm{GHz}, 143.18 \mathrm{GHz}$, and $143.25 \mathrm{GHz}$, respectively. We infer that the $f_{\mathrm{r}}$ should be very near to $0.143 \mathrm{THz}$.
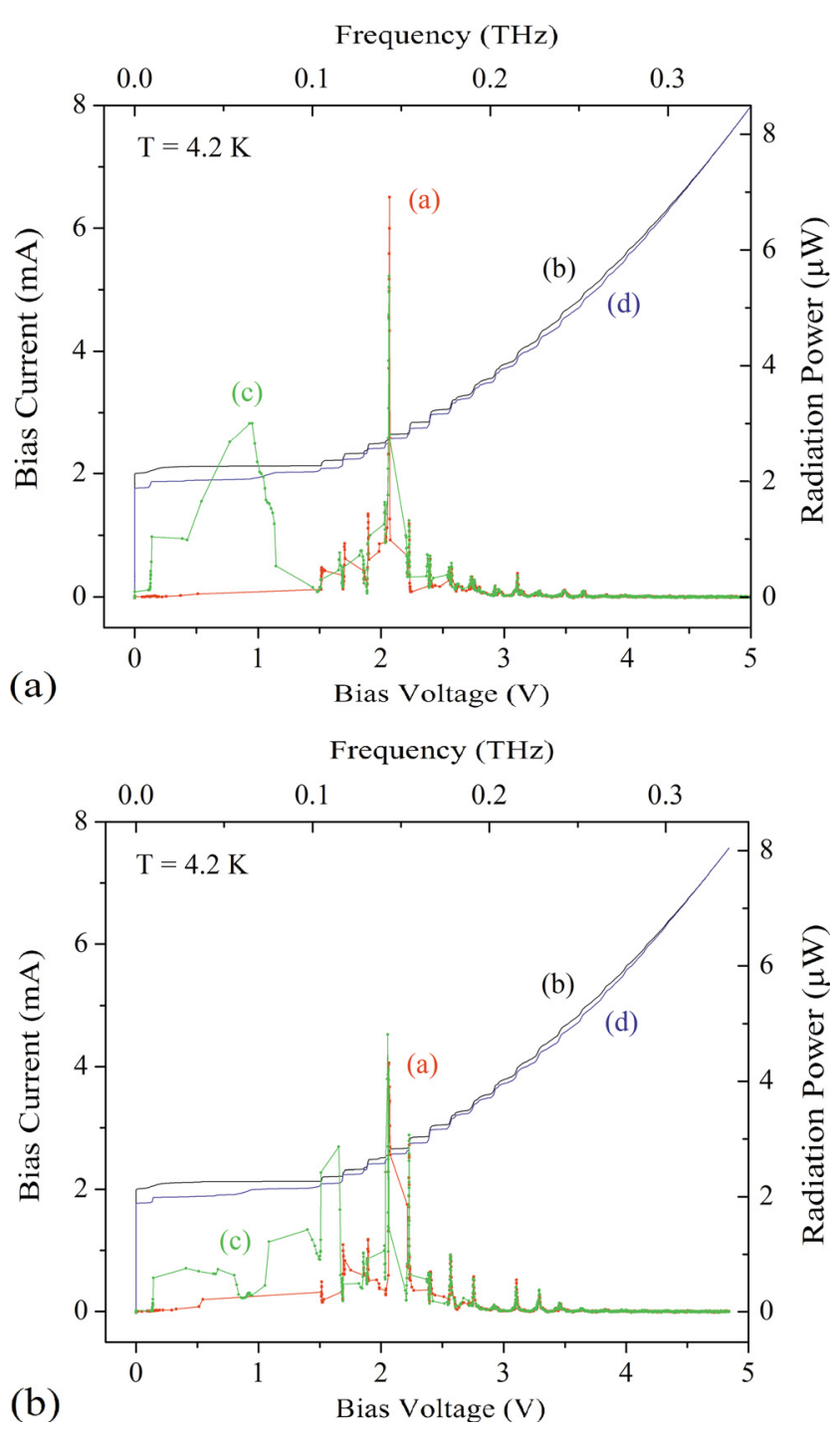

FIG. 2. (Color online) Typical $I-V$ curves of the junction array and sub-THz radiation peaks. (a) The detected power [curve (a)] measured with the bias current sweeping up [curve (b)]; the detected power [curve (c)] measured with the bias current sweeping down [curve (d)]. (b) By changing the parameters of the external quasioptical resonator, the amplitude of the radiation peaks at frequencies higher than $0.15 \mathrm{THz}$ increases.

The tunability was limited by the width of the selfinduced step, which equaled to $0.5 \mathrm{GHz}$ approximately [Fig. 3(a)]. However, the step-tunability covering the frequency band of more than $0.15 \mathrm{THz}$ was realized by biasing the junctions on different self-induced steps of the $I-V$ curve (Fig. 2).

Around $0.143 \mathrm{THz}$ we measured the non-Josephson radiation power from all the possible combinations of the seven subarrays connected in series. The radiation was coherent, since the detected power approximately followed the square of the number of biased junctions [Fig. 3(b)]. ${ }^{5}$ There is reason to believe that all the junctions in the large array were synchronized to the excited resonance of their substrate.

The computer simulation of the electromagnetic field distribution on the substrate ${ }^{6}$ was performed to reveal the correlation of radiation peaks on self-induced steps with resonant modes of the substrate cavity. As shown in Fig. 4, a series of resonant modes at different frequencies can be excited by the junction array at different bias voltages $V$, which 

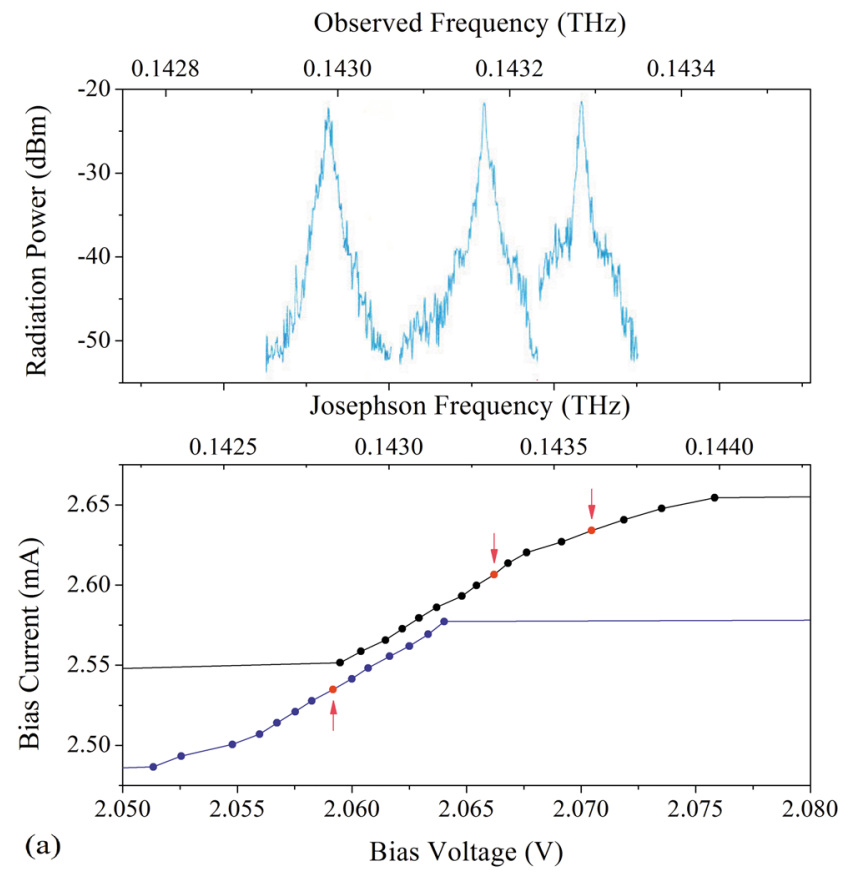

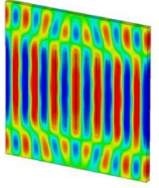

(a)

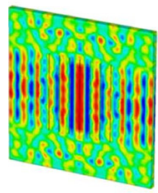

(f)

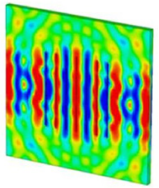

(b)

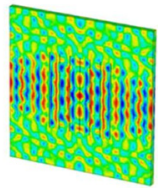

(g)

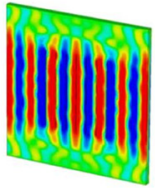

(c)

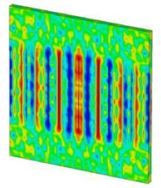

(h)

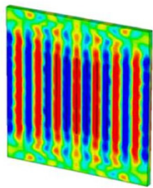

(d)

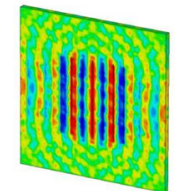

(i)

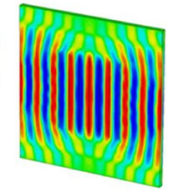

(e)

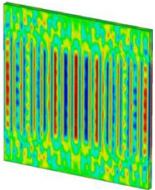

(j)

FIG. 4. (Color online) Electric field distributions of resonant modes on the surface of silicon substrate cavity excited by the large array of Josephson junctions. The resonant modes at frequencies $0.088,0.131,0.143,0.155$, $0.166,0.176,0.190,0.230,0.241$, and $0.252 \mathrm{THz}$ are labeled from (a)-(j).

pling this coherent tunable non-Josephson oscillation into the open space. The maximum power measured at room temperature reached $7 \mu \mathrm{W}$ around $0.143 \mathrm{THz}$. The radiation of available power can be shifted to higher frequencies by optimizing the dielectric constant and dimensions of the substrate, as well as increasing the characteristic frequencies of junctions. While the operation frequency of niobium junctions is limited by the energy gap to around $0.7 \mathrm{THz}$, this restriction may be overcome by using niobium nitride junctions to cover the frequency range up to $1.4 \mathrm{THz} .^{16,17}$

The author Fengbin Song is supported in part by the Project 863 of China under Grant No. 2009AA03Z208. The authors wish to thank A. Braginski for a careful reading of the paper and useful suggestions.

FIG. 3. (Color online) Tunable and coherent non-Josephson radiation around $0.143 \mathrm{THz}$. (a) Three bias points marked by arrows were chosen to observe the radiation peaks on the spectrum analyzer after a heterodyne receiver, which shows that the radiation on the self-induced step is tunable. (b) Radiation power as the function of the square of the number of biased junctions.

qualitatively agrees with our experiments. The excited resonances of the substrate synchronize Josephson junctions in the array, but result in the deviation of radiation peaks from the Josephson frequencies $f_{\mathrm{J}}$. For example, the maximum radiation peak around $0.143 \mathrm{THz}$ originates from the resonant mode of Fig. 4(c) and the peak around $0.155 \mathrm{THz}$ from the mode of Fig. 4(d). At frequencies higher than $0.25 \mathrm{THz}$, the resonant modes of the present substrate become more complicated, and thus unsuitable for synchronizing the junctions and coupling the radiation to the open space. However, this issue can be solved by decreasing the thickness or the dielectric constant of our substrate.

In conclusion, Josephson junctions in the large series array were synchronized by utilizing the resonances excited in their substrate. The sub-THz non-Josephson oscillation near each $f_{\mathrm{r}}$ was pumped by the Josephson oscillation of junctions biased on the corresponding self-induced step. The substrate served also as the dielectric resonator antenna cou-

${ }^{1}$ B. D. Josephson, Phys. Lett. 1, 251 (1962).

${ }^{2}$ B. D. Josephson, Rev. Mod. Phys. 36, 216 (1964).

${ }^{3}$ P. A. A. Booi and S. P. Benz, Appl. Phys. Lett. 64, 2163 (1994).

${ }^{4}$ L. Ozyuzer, A. E. Koshelev, C. Kurter, N. Gopalsami, Q. Li, M. Tachiki, K. Kadowaki, T. Yamamoto, H. Minami, H. Yamaguchi, T. Tachiki, K. E. Gray, W. K. Kwok, and U. Welp, Science 318, 1291 (2007).

${ }^{5}$ A. K. Jain, K. K. Likharev, J. E. Lukens, and J. E. Sauvageau, Phys. Rep. 109, 309 (1984).

${ }^{6}$ F. Song, F. Müller, R. Behr, and A. M. Klushin, Appl. Phys. Lett. 95, 172501 (2009).

${ }^{7}$ F. Song, M. He, M. I. Faley, L. Fang, and A. M. Klushin, J. Appl. Phys. 108, 063903 (2010).

${ }^{8}$ P. E. Lindelof, Rep. Prog. Phys. 44, 949 (1981)

${ }^{9}$ A. H. Dayem and C. C. Grimes, Appl. Phys. Lett. 9, 47 (1966).

${ }^{10}$ F. Auracher and T. Van Duzer, J. Appl. Phys. 44, 848 (1973).

${ }^{11}$ H. Kanter, Appl. Phys. Lett. 23, 350 (1973).

${ }^{12}$ H. Kanter and A. H. Silver, Appl. Phys. Lett. 19, 515 (1971).

${ }^{13}$ F. Mueller, R. Behr, T. Weimann, L. Palafox, D. Olaya, P. D. Dresselhaus, and S. P. Benz, IEEE Trans. Appl. Supercond. 19, 981 (2009).

${ }^{14}$ D. Olaya, P. D. Dresselhaus, S. P. Benz, A. Herr, Q. P. Herr, A. G. Ioannidis, D. L. Miller, and A. W. Kleinsasser, Appl. Phys. Lett. 96, 213510 (2010).

${ }^{15}$ A. Semenov, O. Cojocari, H. W. Hubers, F. Song, A. Klushin, and A. S. Muller, IEEE Electron Device Lett. 31, 674 (2010).

${ }^{16}$ R. Monaco, Int. J. Infrared Millim. Waves 11, 533 (1990).

${ }^{17}$ H. Yamamori, M. Ishizaki, A. Shoji, P. D. Dresselhaus, and S. P. Benz, Appl. Phys. Lett. 88, 042503 (2006). 